\title{
Comparison of Various Cloud Simulation tools available in Cloud Computing
}

\author{
Utkal Sinha $^{1}$, Mayank Shekhar ${ }^{2}$ \\ M.Tech, Computer Science and Engineering, NIT Rourkela, Rourkela, India ${ }^{1}$ \\ M.Tech, Computer Science and Engineering, Tezpur Central University, Tezpur, India ${ }^{2}$
}

\begin{abstract}
Cloud computing is sharing of computer hardware and software resources over the internet so that anyone who is connected to the internet can access it as a service in a seamless way. As we are moving more and more towards the application of this newly emerging technology, it is essential to study, evaluate and analyse the performance, security and other related problems that might be encountered in cloud computing. Since, it is not a feasible way to directly analyse the behaviour of cloud on such problems using the real hardware and software resources due to its high costs, modelling and simulation has become a very powerful tool to cope with these issues. In this paper, we review, analyse and compare features of the existing cloud computing modelling and simulation tools.
\end{abstract}

Keywords: Cloud computing simulators, CloudSim, cloud computing simulators comparison,SPECI, GreenCloud, OCT, Open Cirrus, GroudSim, NetworkCloudSim, EMUSIM, DCSim, iCanCloud, CDOSim, TeachCloud, MDCSim, GDCSim.

\section{INTRODUCTION}

Cloud computing is a new and emerging technology where hosting, content delivery and other real time data the computer hardware and software that we use on our processing. Quantifying the performance of various desktop is shared over the internet and we could access scheduling and allocation policies in a real cloud such computing facilities typically by purchasing it as a computing environment for different application models is service from another company. This sharing of computing extremely challenging and costly. Thus we go for cloud is being made possible with the extensive use simulation tools to analyse the performance of a Cloud virtualization technologies and distributed computing. The system with reduced complexity.

Cloud computing providers offer their cloud services based on several fundamental models as follows:

\section{BACKGROUND}

- Infrastructure as a service (IaaS):

According to the IETF (Internet Engineering Task Force), the providers of IaaS services offer virtual machines and other resources. The cloud user have to install the operating system images in order to deploy their cloud applications.

\section{- Platform as a service (PaaS):}

An extensive study on the various available Cloud simulators have been made. The list of Cloud simulators that we have encountered are: CloudSim, CloudAnalyst, SPECI, Green-Cloud, OCT(Open Closed Testbed), Open Cirrus, GroudSim, Network CloudSim, EMUSIM, DCSim, iCanCloud, GDCSIm, MDCSim, CDOSim, TeachCloud.

\section{III.CLOUD SIMULATION TOOLS}

In this model, the cloud provider delivers a computing platform. It includes the operating system, programming language execution environment, database and web server.

- Software as a service (SaaS):

Cloud providers in this model provides access to the application software and databases to the users.

- Unified Communications as a Service (UCaaS):

In the UCaaS model, multi-platform communications over the network are packaged by the service provider. The services could be in different devices, such as computers and mobile devices.

People are migrating from the traditional computing towards cloud computing because it is agile, cost reductions, device and location independence, higher reliability, fault tolerance etc. Some of the cloud based application services include social networking, web

In this section, we provide an overview of the various cloud computing simulators which are used to evaluate the performance and security of cloud computing systems, and describe their main.

\section{A. CloudSim}

CloudSim is a toolkit (library) for simulation of Cloud computing environments developed in the CLOUDS Laboratory at the Computer Science and Engineering Department of the University of Melbourne, Australia. It provides basic classes for describing data centers, virtual machines, applications, users, computational resources, and policies for management of diverse parts of the system (e.g., scheduling and provisioning). These components can be put together for users to evaluate new policies, schedulingalgorithms, mapping etc. in Cloud. It is a complex simulation toolkit using which most of the Cloud scenarios can be built by simply extending or replacing the classes and coding the desired scenario. 
It is important to note that CloudSim is not a ready-to-use solution where you set parameters and collect results for use in your project. Instead, being a library, CloudSim requires that we write a Java program using its compose the desired scenario and collect well as optimization ofcommunication protocols and the results for the analysis of performance and security of network infrastructures.

Cloud applications.

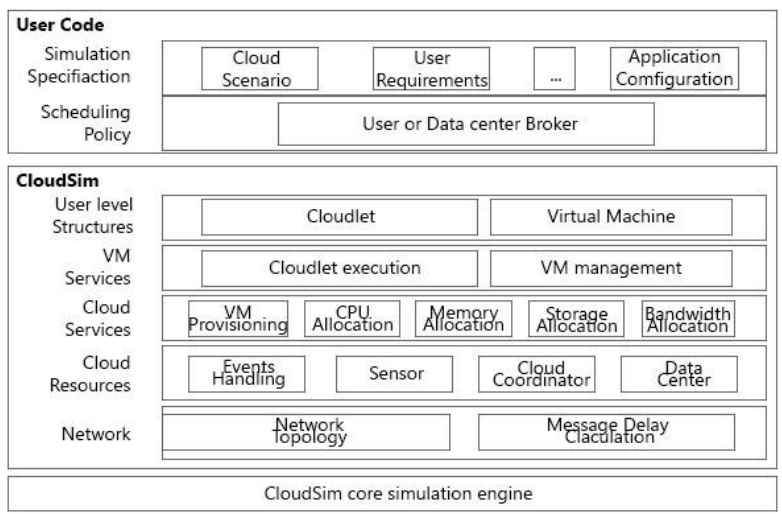

Figure 1: Layered CloudSim Architecture

All the components in CloudSim communicate with each other through message passing. In the above layered architecture of CloudSim, the lowest layer is mainly responsible for communication between the components and the second layer has all the sub layers in it that have the main Cloud components like sensors, data centres etc. [1]. Using CloudSim a user can model data centre, virtual machine allocation using a VMScheduler, power consumption and network behaviour.

Other simulation tools that extends the power of CloudSim are: CloudSimEx, WorkflowSim, SimpleWorkflow, RealCloudSim, CloudReports, CloudAuction, CloudMIG Xpress, CloudAnalyst [2].

\section{B. SPECI}

According to [3], SPECI, (Simulation Program for Elastic Cloud Infrastructures), is a simulation tool which allows exploration of aspects of scaling as well as performance properties of future datacentres. Given the size and middleware design policy as the input, SPECI simulates the performance and behaviour of data centres.

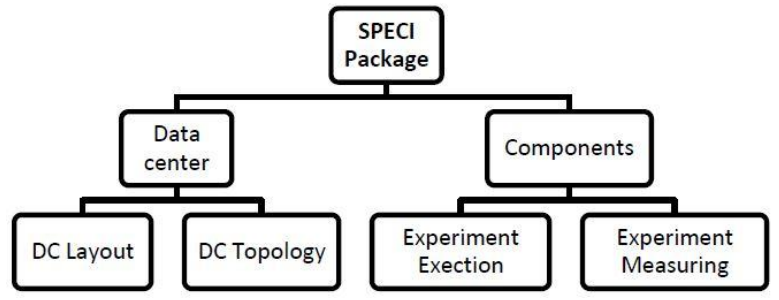

Figure 2: SPECI Package

\section{GreenCloud}

GreenCloud, as proposed by Kliazovich, is a sophisticated packet-level simulator for energy-aware cloud computing data centres with a focus on cloud communications. It offers a detailed fine-grained modelling of the energy consumed by the data centre IT equipment, such as computing servers, network switches, and communication links. It can be used to develop novel solutions in monitoring, resource allocation, workload scheduling as

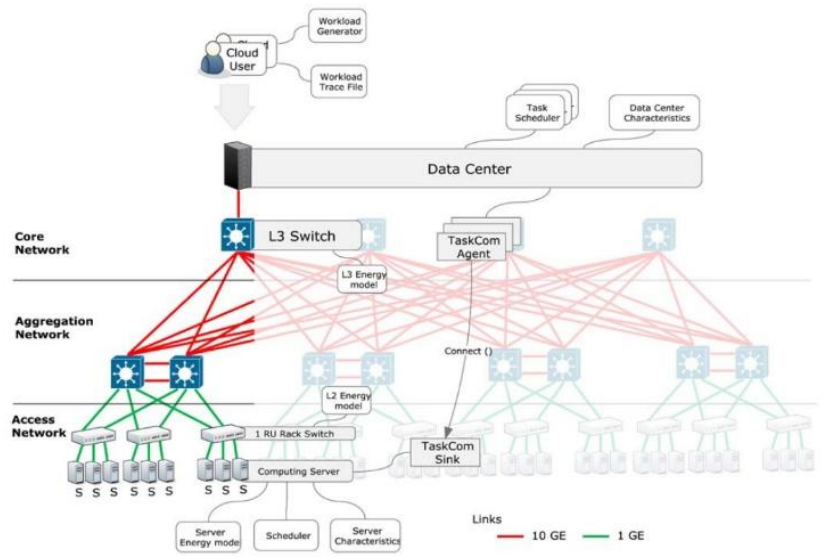

Figure 3: Architecture of the GreenCloud simulation environment

Open Cloud Testbed (OCT) was proposed by Grossman et al. [5] to mainly benchmark different cloud computing systems, to investigate their interoperability. It is currently configured as a smaller-scale testbed. It is use to develop cloud computing software and infrastructure.

The architecture of OCT is shown in the below Figure 4:

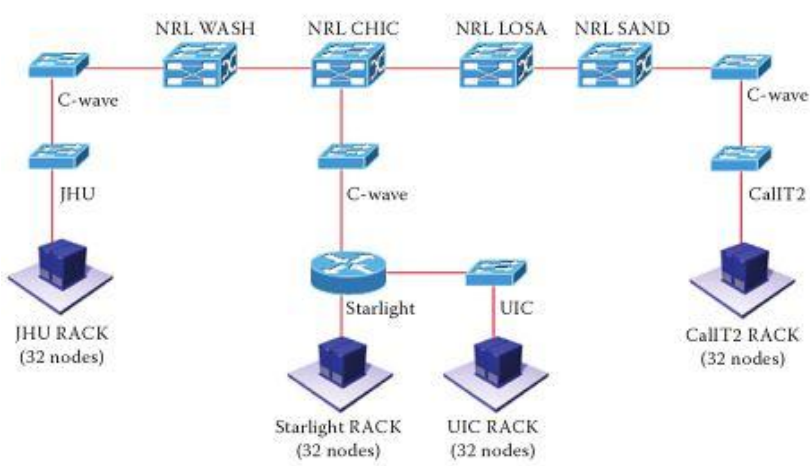

Figure 4: Architecture of the OCT Cloud simulator

The main characteristics of OCT [5] are as follows:

- Different cloud systems and services are installed, including Eucalyptus, Hadoop, CloudStore (KosmosFS), Sector/Sphere, and Thrift in order to make OCT easier to study interoperability and benchmarking, to develop network libraries, monitoring systems and benchmarking suites to support the development and experimental studies of cloud computing stacks.

- The architecture of OCT includes high-performance protocols, services, and infrastructure at all levels. It uses a high-performance $10 \mathrm{~Gb} / \mathrm{s}$ network based on extremely fast transport protocols supported by dedicated light paths instead of using the internet.

\section{OCT ( Open Cloud Testbed)}


The OCT infrasreucture consists of 4 data centers located which allows more accurate evaluation of scheduling and at John Hopkins University (Baltimore), Startlight resource provisioning policies to optimize the performance (Chicago), The University of Illinois (Chicago), and the of a Cloud infrastructure.

University of California (San Diego). Each rack has 32 nodes. Each node has dual dual-core AMD $2.4 \mathrm{GHz} \mathrm{CPU}$, 12GB memory, 1TB single SATA disk, and dual 1GE NICs. Two Cisco $3750 \mathrm{E}$ switches connect the 32 nodes, which then connects to the outside by a $10 \mathrm{~Gb} / \mathrm{s}$ uplink.

\section{E. Open Cirrus}

Open Cirrus is an open cloud computing testbed sponsored by Hewlett-Packard (HP), Inter, and Yahoo! in collaboration with many other organizations. According to [6] the Open Cirrus aspires to achieve the below goals:

- Foster systems-level research in cloud computing.

- Encourage new cloud computing applications and applications-level research.

- Offer a collection of experimental datasets.

- Develop open-source stacks and APIs for the cloud.

Open Cirrus offers a cloud stack consisting of physical and virtual machines, and global services, such as sign-on, monitoring, storage, and job submission. A platform for real world applications and services.

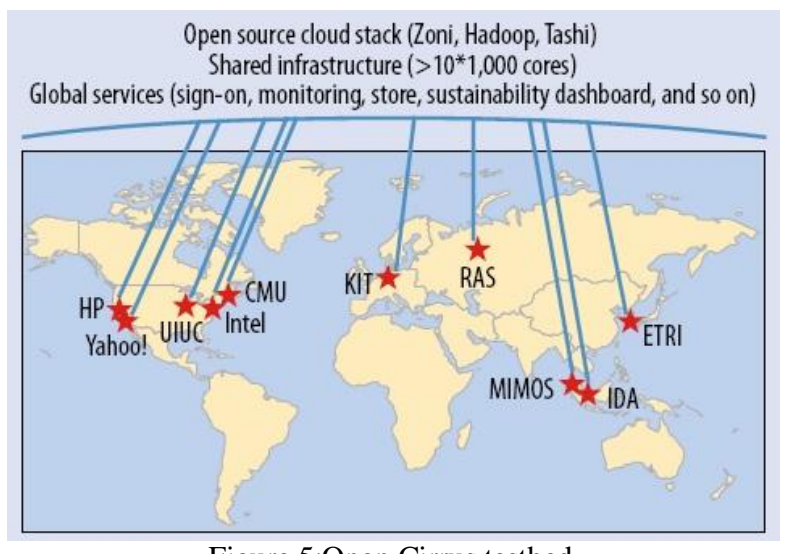

Figure 5:Open Cirrus testbed

\section{F. GroudSim}

GroudSim is a is an event based simulator proposed by Ostermann et al. [7]for scientific applications on Grid and Cloud environments based on a scalable simulationindependent discrete-event core. It provides a comprehensive set of features for complex simulation scenarios from simple job executions on leased computing resources to calculation of costs, and background load on resources. Simulations can be parameterised and are easily extendable by probability distribution packages for failures which normally occur in complex environments. It is mainly concentrated on the IaaS, but it is easily extendable to support additional models such as PaaS, DaaS and TaaS [8].

\section{G. NetworkCloudSim}

NetworkCloudSim, as proposed by S.K. Garg and RajkumarBuyya [9], is an extension of CloudSim with a scalable network and generalized application model,

\section{H. EMUSIM}

EMUSIM (Integrated Emulation And Simulation) combines emulation (AEF) and simulation (CloudSim) [10] to enable more accurate models of software artefacts (obtained via profiling during emulation) to be used during simulations. This is especially useful when the tester has no idea on the performance of the software under different levels of concurrency and parallelism, which impedes utilization of simulation.

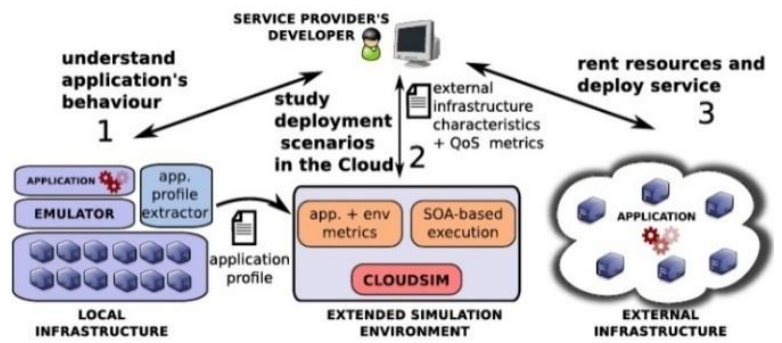

Figure 6: Internal organization of EMUSIM

\section{DCSim}

DCSim, proposed by [11] is an extensible simulation framework for simulating a data centre hosting an Infrastructure as a Service cloud.

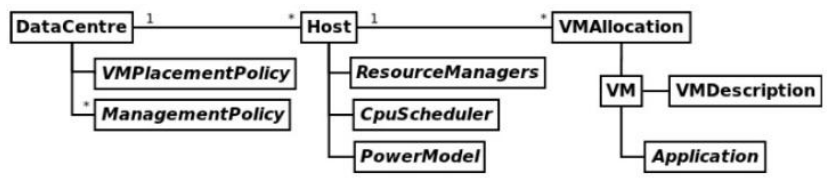

Figure 7: DCSim architecture

\section{J. iCanCloud}

iCanCloud is another tool for simulating high performance MPI applications on large storage networks [12].This simulator is developed over SIMCAN (a simulation tool to analyze high performance I/O architectures).

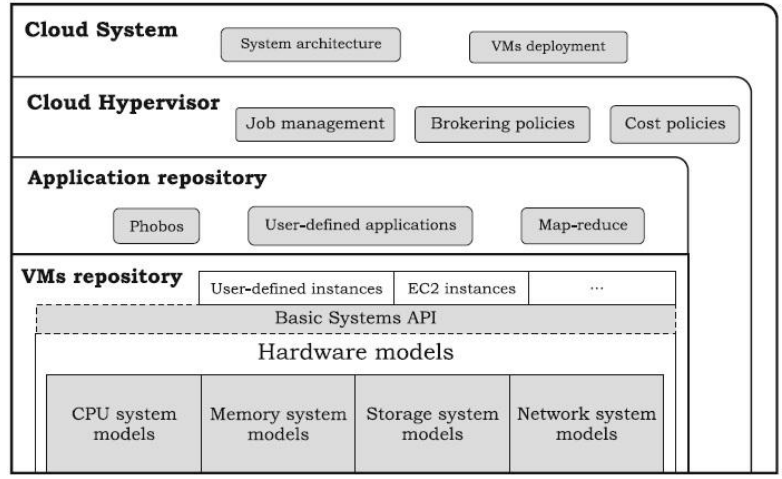

Figure 8: Layered architecture of iCanCloud

In this simulator, there is no need to modify the simulator code to test different architectures. It can be done just by creating a new configuration file. 


\section{K. CDOSim}

CDOSim is a cloud deployment option (CDO) Simulator which can simulate the response times, SLA violations and costs of a CDO. A CDO is a decisions concerning simulator which takes decision about the selection of a cloud provider, specific runtime adaptation strategies, components deployment of virtual machine and its instances configuration. Component deployment to virtual machine instances includes the possibility of forming new components of already existing components. Virtual machine instances configuration, refer to the instance type of virtual machine instances. CDOSim can simulate cloud deployments of software systems that were reverse engineered to KDM models. CDOSim has ability to represent the users rather than the providers perspective. CDOSim is a simulator that allows the integration of finegrained models. CDOSim is best example for comparing runtime reconfiguration plans or for determining the tradeoff between costs and performance [13]. CDOSim is designed to address the major shortcomings of other existing cloud simulators such as

- Consequently oriented towards the cloud user perspective instead of exposing fine-grained internals of a cloud platform.

- Mitigates the cloud users lack of knowledge and control concerning a cloud platform structure

- Simulation is independent of concrete programming languages in the case appropriate $\mathrm{KDM}$ extractors exist for a particular language.

- Workload profiles from production monitoring data can be used to replay actual user behavior for simulating CDOs.

\section{TeachCloud}

TeachCloud [14] is a modeling and simulation environment for cloud computing. Students can use TeachCloud to experiment with different cloud components such as: processing elements, data centers, networking, Service Level Agreement (SLA) constraints,web-based applications, Service Oriented Architecture (SOA), virtualization, management and automation, and Business Process Management (BPM). TeachCloud is an extension of CloudSim, a researchoriented simulator used for the development and validation in cloud computing.

\section{MDCSim}

MDCSim is a variant of CloudSim tools. It helps the user to analyze and predict the hardware related parameters of the data centers like those of servers, switches, routers etc. Also it is used predominantly because of its low overhead produced [8].

\section{N. GDCSim}

Green Data Center Simulator [15] (GDCSim) is a simulator for studying the energy efficiency of data centers under various data center geometries, workload characteristics, platform power management schemes, and scheduling algorithms. GDCSim is used to iteratively design green data centers. It is suitable for online analysis.

\section{IV.COMPARISON OF CLOUD COMPUTING SIMULATORS}

A brief comparison among the various cloud simulators is shown in the below table

\begin{tabular}{|c|c|c|c|c|c|c|}
\hline $\begin{array}{c}\text { CLOUD } \\
\text { SIMULATORS }\end{array}$ & $\begin{array}{c}\text { GUI } \\
\text { SUPPORT }\end{array}$ & PLATFORM & LANGUAGE & $\begin{array}{c}\text { SUPPORT OF } \\
\text { TCP/IP }\end{array}$ & $\begin{array}{l}s / W \text { or } \\
H / W\end{array}$ & AVAILIBILITY \\
\hline Cloudsim & $\begin{array}{l}\text { Limited } \\
\text { (CloudAnalyst) }\end{array}$ & Gridsim & IAVA & None & s/w & $\begin{array}{l}\text { Open Source } \\
\text { (hetp://www.eloudbus. } \\
\text { org/cloudsim) }\end{array}$ \\
\hline SPECI & Limited & SimKit & IAVA & None & $s, \mathrm{~W}$ & $\begin{array}{l}\text { Open Source } \\
\text { (http://wwww.speci.org/ } \\
\text { ) }\end{array}$ \\
\hline Greencloud & $\begin{array}{l}\text { Limited GUI } \\
\text { support (Via } \\
\text { Nam) }\end{array}$ & NS 2 & $\mathrm{C}+\mathrm{O}, \mathrm{Otel}$ & $\begin{array}{l}\text { Full TCP/IP } \\
\text { protocol }\end{array}$ & $s / N$ & $\begin{array}{l}\text { Open Source } \\
\text { (http://greencloud_gfor } \\
\text { ge.uni.lu) }\end{array}$ \\
\hline $\begin{array}{l}\text { OCT (Open Cloud } \\
\text { Testbed) }\end{array}$ & $\begin{array}{l}\text { Limited (Via } \\
\text { Open Cloud } \\
\text { Testbed } \\
\text { Monitor) }\end{array}$ & $\begin{array}{l}\text { Geographically } \\
\text { distributed cloud } \\
\text { testbed spanning } \\
\text { four data centers }\end{array}$ & NA & NA & $\begin{array}{l}\text { Both } S / W \text { and } \\
H / W W\end{array}$ & $\begin{array}{l}\text { Limited (Registration } \\
\text { is needed) }\end{array}$ \\
\hline Open Cirrus & None & $\begin{array}{l}\text { Federation of } \\
\text { heterogenous } \\
\text { data centers }\end{array}$ & NA & NA & $\begin{array}{l}\text { Both } S / W \text { and } \\
\mathrm{H} / \mathrm{W}\end{array}$ & $\begin{array}{l}\text { Open Source } \\
\text { (http://opencirrus.inte } \\
\text { 1-research.net/) }\end{array}$ \\
\hline Groudsim & None & NA & IAVA & $\begin{array}{l}\text { Full TCP/IP } \\
\text { protocol }\end{array}$ & $s / N$ & NA \\
\hline NetworkCloudsim & None & Cloudsim & IAVA & None & s/W & $\begin{array}{l}\text { Open Source } \\
\text { (http://wwww.cloudbus. } \\
\text { org/cloudsim) }\end{array}$ \\
\hline
\end{tabular}


International Journal of Advanced Research in Computer and Communication Engineering Vol. 4, Issue 3, March 2015

\begin{tabular}{|c|c|c|c|c|c|c|}
\hline EMUSIM & $\begin{array}{l}\text { Limited (Via } \\
\text { NEPI) }\end{array}$ & AEF, CloudSim & JAVA & None & $\$ / W$ & $\begin{array}{l}\text { Open Source } \\
\text { (http://www.cloudbus. } \\
\text { org/cloudsim/emusim) }\end{array}$ \\
\hline DCSim & None & NA & JAVA & $\begin{array}{l}\text { No TCP/IP } \\
\text { Support }\end{array}$ & $\$ / W$ & $\begin{array}{l}\text { Open Source } \\
\text { (https://github.com/di } \\
\text { gs-uwo/dcsim) }\end{array}$ \\
\hline iCanCloud & GUI Supports & SIMCAN & $\begin{array}{l}\text { OMNet, MPL, } \\
\mathrm{C}++\end{array}$ & None & $s / W$ & $\begin{array}{l}\text { Open Source } \\
\text { (http://www.arcos.inf. } \\
\text { uc3m.es/ icancloud/d } \\
\text { ownloads, html) }\end{array}$ \\
\hline cDosim & None & Cloudsim & JAVA & $\begin{array}{l}\text { No TCP/IP } \\
\text { Support }\end{array}$ & $\$ / W$ & NA \\
\hline TeachCloud & Full GUI toolkit & Cloudsim & NA & None & $\$ / W$ & $\begin{array}{l}\text { Open Source } \\
\text { (https://code.google.co } \\
\mathrm{m} / \mathrm{p} / \text { teachcloud/sourc } \\
\text { e) }\end{array}$ \\
\hline MDCsim & No GUI Support & $\operatorname{csim}$ & $C+/$ JAVA & None & S/W & Commercial \\
\hline GDCSim & No GUI Support & BlueTool & $\mathrm{CH} / \mathrm{XML}$ & None & $\$ / W$ & $\begin{array}{l}\text { Open Source } \\
\text { (https://impact.asu.ed } \\
\text { u/BlueTool/wiki/index. } \\
\text { nhn/AlueTnnll }\end{array}$ \\
\hline
\end{tabular}

Table 1: Comparison between Cloud Simulators

\section{CONCLUSION}

In this report, we have projected numerous cloud simulation tools. We have also shown there properties with respect to their TCP/IP support, GUI Support, under lying platform and the programming language. In general, every simulator has its pros and cons. So, we need to choose the appropriate simulator depending on the user requirement.

\section{REFERENCES}

[1] A. K. R. Parveen Kumar, "An overview and survey of various cloud simulation tools," in Journal of Global Research in Computer Science, vol. 5, no. 1, January 2014.

[2] [Online].Available: http://www.cloudbus.org/cloudsim/.

[3] I. Sriram, "Speci, a simulation tool exploring cloud-scale data centres," in CloudCom 2009, LNCS 5931, pp. 381-392, vol. 5, no. 1, January 2014.

[4] [Online]. Available: http://www.isi.edu/nsnam/ns/

[5] C. B. J. S. J. M. Robert Grossman, YunhongGu, "The open cloud testbed: a wide area testbed for cloud computing utilizing hign performance network services," in GridNets 2009, 2009.

[6] M. H. S. Y. K. M. K. Roy Cambpell, Indranil Gupta, "Open cirrus cloud computing testbed: Federated data centers for open source systems and services research," in The USENIX Hotcloud 2009, 2009.

[7] R. P. T. F. S. Ostermann, K. Plankensteiner, "Groudsim: an eventbased simulation framework for computational grids and clouds," in CoreGRID/ERCIM Workshop on Grids and Clouds. Springer Computer Science Editorial, Ischia, 2010. 2010.

[8] D. R. M. P. Jain;, "Study and comparison of various cloud simulators available in the cloud computing," in International Journal of Advanced Research in Computer Science and Software Engineering, vol. 3, no. 9, September 2013.
[9] R. B. Saurabh Kumar Garg, "Networkcloudsim: Modelling parallel applications in cloud simulations," in Fourth IEEE International Conference on Utility and Cloud Computing, 2011, 2011.

[10] J. GUSTEDT, E. JEANNOT, and M. QUINSON, "Experimental methodologies for large-scale systems: A survey," vol. 19, no. 03, 2009, pp. 399-418. [Online]. Available: http://www.worldscientific.com/doi/abs/10.1142/S0129626409000304

[11] M. Tighe, G. Keller, M. Bauer, and H. Lutfiyya, "Dcsim: A data centre simulation tool for evaluating dynamic virtualized resource management," in Network and service management (cnsm), 2012 8th international conference and 2012 workshop on systems virtualiztion management (svm), Oct 2012, pp. 385-392.

[12] A. Nunez, J. Fernandez, J. Garcia, and J. Carretero, "New techniques for simulating high performance mpi applications on large storage networks," in Cluster Computing, 2008 IEEE International Conference, Sept 2008, pp. 444-452.

[13] W. H. Sren Frey, Florian Fittkau, "Cloud user-centric enhancements of the simulator cloudsim to improve cloud deployment option analysis," in ESOCC'12 Proceedings of the First European conference on Service- Oriented and Cloud Computing, Sept 2012, pp. 200-207.

[14] Y. Jararweh, M. J. Z. Alshara, and M. N. A. M. Kharbutli, "Teachcloud: A cloud computing educational toolkit," in Proceedings of the 1st International IBM Cloud Academy Conference (ICA CON 2012), IBM, Research Triangle Park, NC, USA, 2012, 2012

[15] S. Gupta, R. Gilbert, A. Banerjee, Z. Abbasi, T. Mukherjee, and G. Varsamopoulos, "Gdcsim: A tool for analyzing green data center design and resource management techniques," in Green Computing Conference and Workshops (IGCC), 2011 International, July 2011, pp. 1-8. 
International Journal of Advanced Research in Computer and Communication Engineering Vol. 4, Issue 3, March 2015

\section{BIOGRAPHIES}

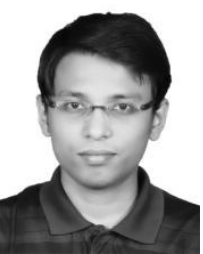

Utkal Sinha is a postgraduate scholar ( M.Tech ) at the National Institute of Technology Rourkela. His area of interest includes cloud computing, image processing and computer vision and machine learning.

Mayank Shekhar received his M.Tech degree from Tezpur Central University, Tezpur. His area of interest includes distributed and cloud computing and wireless sensor networks. 